\title{
Medidas de recuperación del sector turístico en América Latina y el Caribe: una oportunidad para promover la sostenibilidad y la resiliencia
}

\section{Resumen}

Desde abril de 2020, el turismo en la región se ha detenido temporalmente como resultado de la pandemia de la enfermedad por coronavirus (COVID-19). Esta parálisis no solo ha afectado duramente a las economías y el empleo del Caribe, sino también a muchas comunidades locales de América Latina. En este informe se examina la contribución del turismo a las exportaciones, el producto interno bruto (PIB) y el empleo, así como la reciente disminución de esta actividad en la región. Un escenario del impacto muestra que la caída del turismo podría llevar a una disminución del crecimiento del PIB total en el Caribe y América Latina de 8 puntos porcentuales y 1 punto porcentual, respectivamente, mientras que el empleo total podría disminuir 9 puntos porcentuales en el Caribe y 2 puntos porcentuales en América Latina. Dado que los países han adoptado medidas para mitigar los efectos de la crisis en el turismo, se formulan recomendaciones para intensificar la preparación del sector para la recuperación económica y, al mismo tiempo, fomentar la diversificación y la sostenibilidad ambiental y social.

\section{A. El papel clave del turismo en las exportaciones, el PIB y el empleo}

La pandemia del coronavirus no solo ha causado un desastre humanitario, sino que también ha paralizado sectores clave como el turismo. Este sector fue uno de los primeros y más afectados de la economía. Según la Organización Mundial del Turismo (OMT, 2020), las llegadas de turistas internacionales pueden disminuir entre un 58\% y un 78\% en 2020. Este sector no solo es uno de los principales contribuyentes a las exportaciones, la economía y el empleo en el Caribe, sino también en muchas ciudades y comunidades locales de América Latina.

El turismo es un generador clave de divisas, ingresos y empleo en toda la región. En 2019, representó el $42 \%$ y el 10\% de las exportaciones totales (bienes y servicios) en el Caribe y en América Latina, respectivamente. Su participación superó el 50\% en algunos países del Caribe (véase el gráfico 1.A). La economía del turismo, que incluye tanto el turismo como todos los sectores que dependen de él, representó el 26\% del PIB total en el Caribe y el 10\% en América Latina. Dado que la economía del turismo es muy intensiva en mano de obra, representó el 35\% del empleo en el Caribe y el 10\% en América Latina (véase el gráfico 1.B). En el Caribe, el sector turístico depende casi totalmente de los visitantes extranjeros. En cambio, los visitantes nacionales representan casi tres cuartas partes del total en México, mientras que en algunos países de América del Sur (como la Argentina, el Brasil, Chile y el Perú) esta proporción es superior al 50\%.

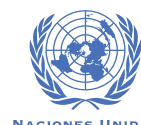

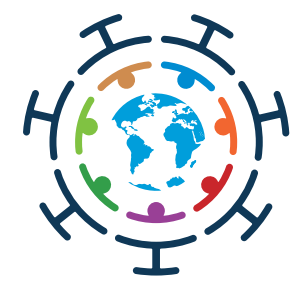

COVID-19 RESPUESTA
Resumen

A. El papel clave del turismo en las exportaciones, el PIB y el empleo

B. La drástica caída del turismo

C. Impactos sustanciales en las exportaciones, el PIB y el empleo

$D$. Diferentes medidas pueden mitigar el impacto de la crisis y acelerar la recuperación

Bibliografía 
Gráfico 1

América Latina y el Caribe (33 países): participación del turismo en las exportaciones, el PIB total y el empleo, 2019 (En porcentajes)

\section{A. Participación del turismo en las exportaciones de bienes y servicios}

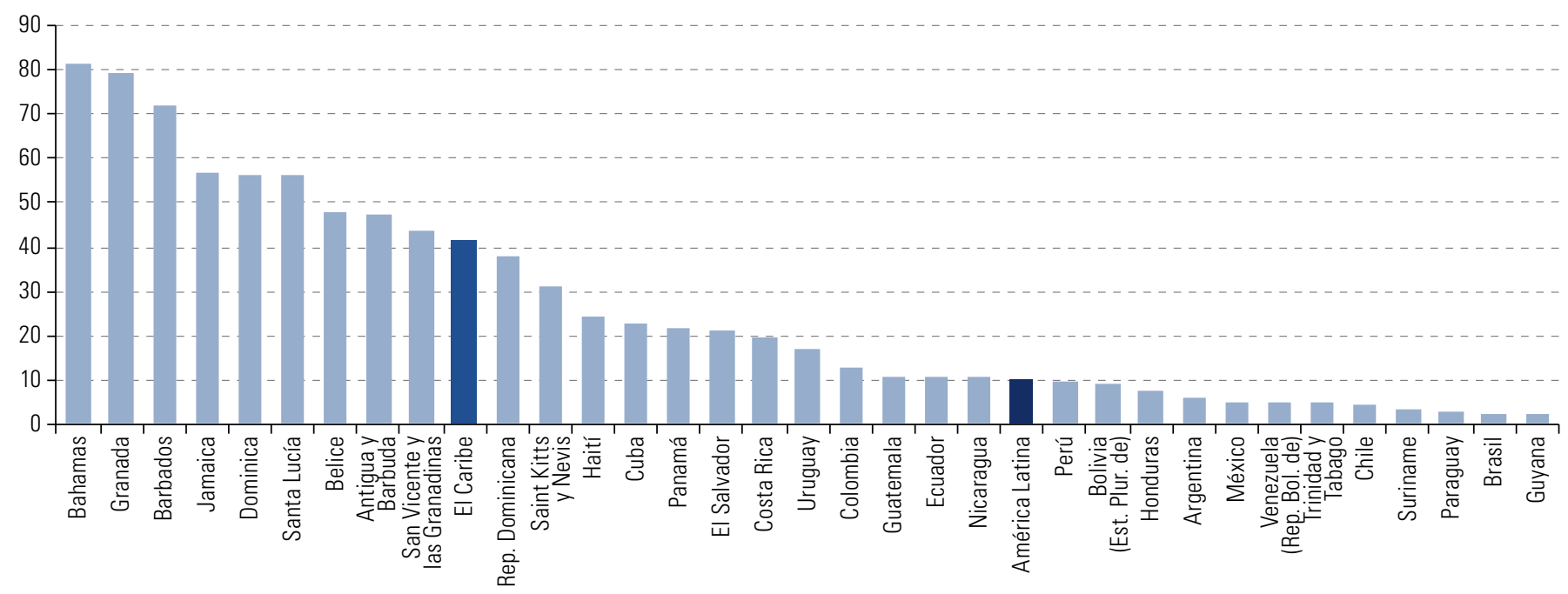

\section{B. Participación de la economía del turismo en el PIB total y el empleo}

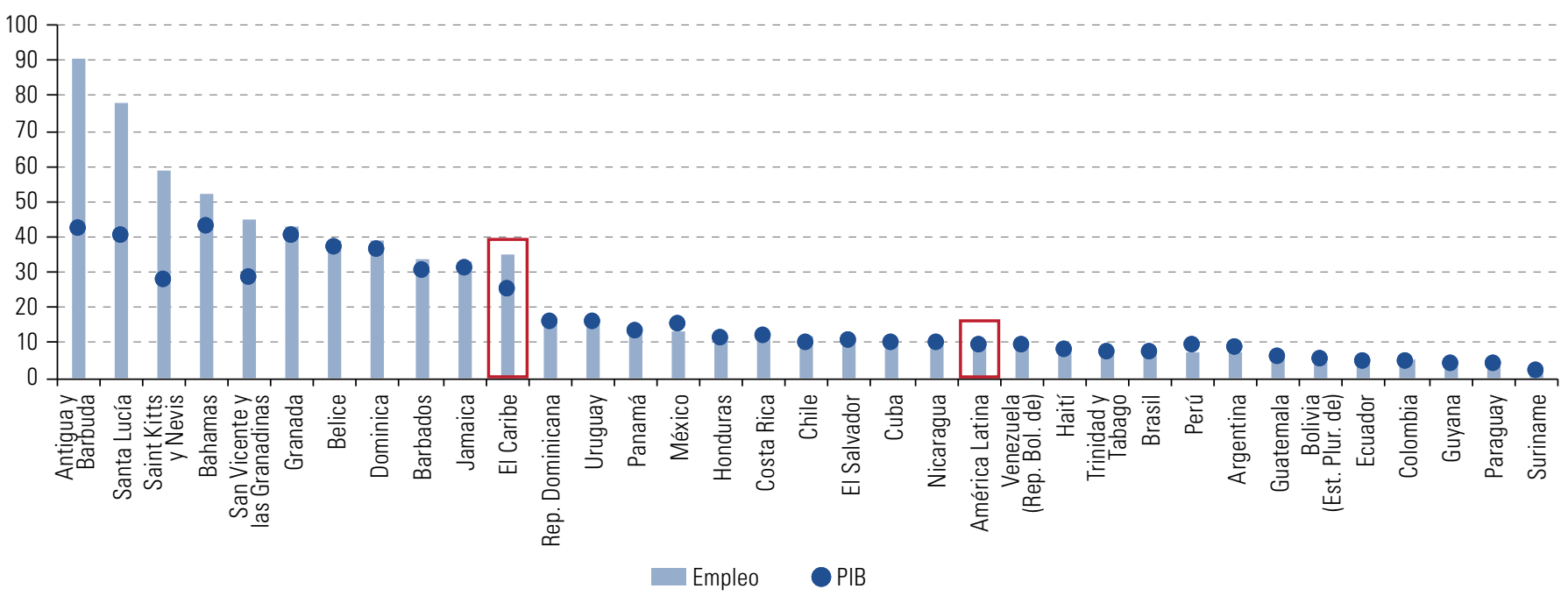

Fuente: Comisión Económica para América Latina y el Caribe (CEPAL), sobre la base de datos del Consejo Mundial de Viajes y Turismo, y Banco Mundial, World Development Indicators [en línea] http://data.worldbank.org/data-catalog/world-development-indicators.

Nota: La economía del turismo incluye tanto el turismo como todos los sectores que dependen de él. Las cifras correspondientes a América Latina y el Caribe son promedios nacionales no ponderados. El Caribe incluye a todos los países miembros de la Comunidad del Caribe (CARICOM) (excepto Montserrat), Cuba y la República Dominicana.

El turismo ofrece oportunidades fundamentales para las mujeres al ofrecerles horarios de trabajo flexibles y empleos a tiempo parcial, aunque las brechas de género siguen existiendo. Las mujeres representan casi el $60 \%$ de los trabajadores en actividades de alojamiento y alimentación en América Latina y el $61 \%$ en el Caribe. Aunque muchas mujeres trabajan en empleos de bajo nivel y mal remunerados (OMT, 2019), la mitad de las empresas turísticas de la región son propiedad de mujeres (Banco Mundial, 2017).

Las micro, pequeñas y medianas empresas (mipymes) representan la mayor parte de las empresas turísticas. Por ejemplo, las mipyme representaban el 98,7\% de las empresas 
relacionadas con el turismo en Costa Rica y el 99,8\% en México (cuenta satélite de turismo, Costa Rica 2016 y México 2018) (BCCR, 2018; INEGI, 2019). Estas relaciones son similares a las del conjunto de la economía latinoamericana, en la que las mipymes representan el 99\% del sistema productivo y el 61\% del empleo (Dini y Stumpo, 2018).

\section{B. La drástica caída del turismo}

La crisis actual del COVID-19 ha causado una importante caída en la actividad turística en todo el mundo, incluida la región de América Latina y el Caribe. Entre 2016 y principios de 2020, las llegadas de turistas internacionales crecieron a un ritmo de alrededor del 10\% anual en México y las tres subregiones. Sin embargo, a medida que la pandemia llegó a la región y un número cada vez mayor de países cerró sus fronteras en marzo, estas llegadas disminuyeron más del $50 \%$ en marzo y cerca del 100\% en abril en las tres subregiones y en México (véase el gráfico 2).

\section{Gráfico 2}

América Latina y el Caribe (subregiones seleccionadas)a : variaciones anuales de las llegadas mensuales de turistas internacionales, enero de 2016 a abril de 2020

(En porcentajes)

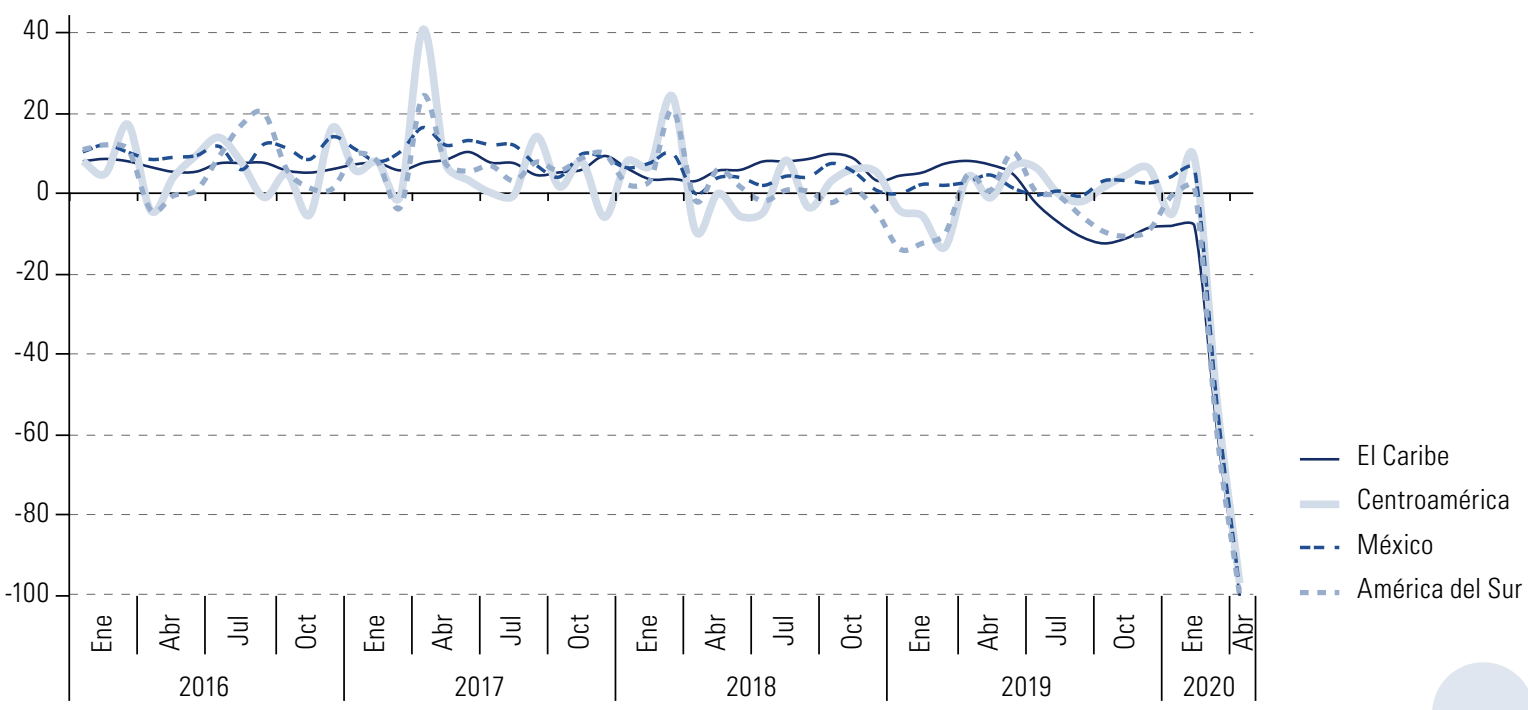

Fuente: Comisión Económica para América Latina y el Caribe (CEPAL), sobre la base de cifras oficiales.

a Las subregiones comprenden los siguientes países con datos mensuales completos: el Caribe: Bahamas, Belice, Cuba Jamaica, la República Dominicana y Santa Lucía; Centroamérica: Costa Rica y Guatemala; América del Sur: Argentina, Chile Ecuador, Perú y Uruguay.

Los vuelos internacionales y nacionales también han disminuido a casi cero. Las salidas diarias de vuelos de los aeropuertos de las diferentes subregiones disminuyeron abruptamente durante la segunda quincena de marzo (véase el gráfico 3), al igual que las llegadas de vuelos. Desde abril, la mayoría de los demás vuelos han sido de carga o con fines humanitarios. Varias de las principales aerolíneas regionales (como Avianca, Caribbean Airlines, Copa, LATAM y LIAT) han buscado apoyo gubernamental ante la fuerte pérdida de ingresos o la quiebra (Antigua News Room, 2020a).

En el sector turístico del Caribe, la industria de los cruceros se ha visto particularmente afectada. Entre 2010 y 2018, este sector creció en promedio un 8\% anual. En 2019, el Caribe recibió el 38\% del total mundial de pasajeros de cruceros y al 34\% del despliegue total de cruceros. Sin embargo, a medida que la pandemia se extendió por todo el mundo, el número de pasajeros se redujo casi a cero a mediados de marzo de 2020 (Panetta, 2020). 
Gráfico 3

América Latina y el Caribe (subregiones seleccionadas): salidas de vuelos diarios, 1 de marzo a 12 de junio de 2020

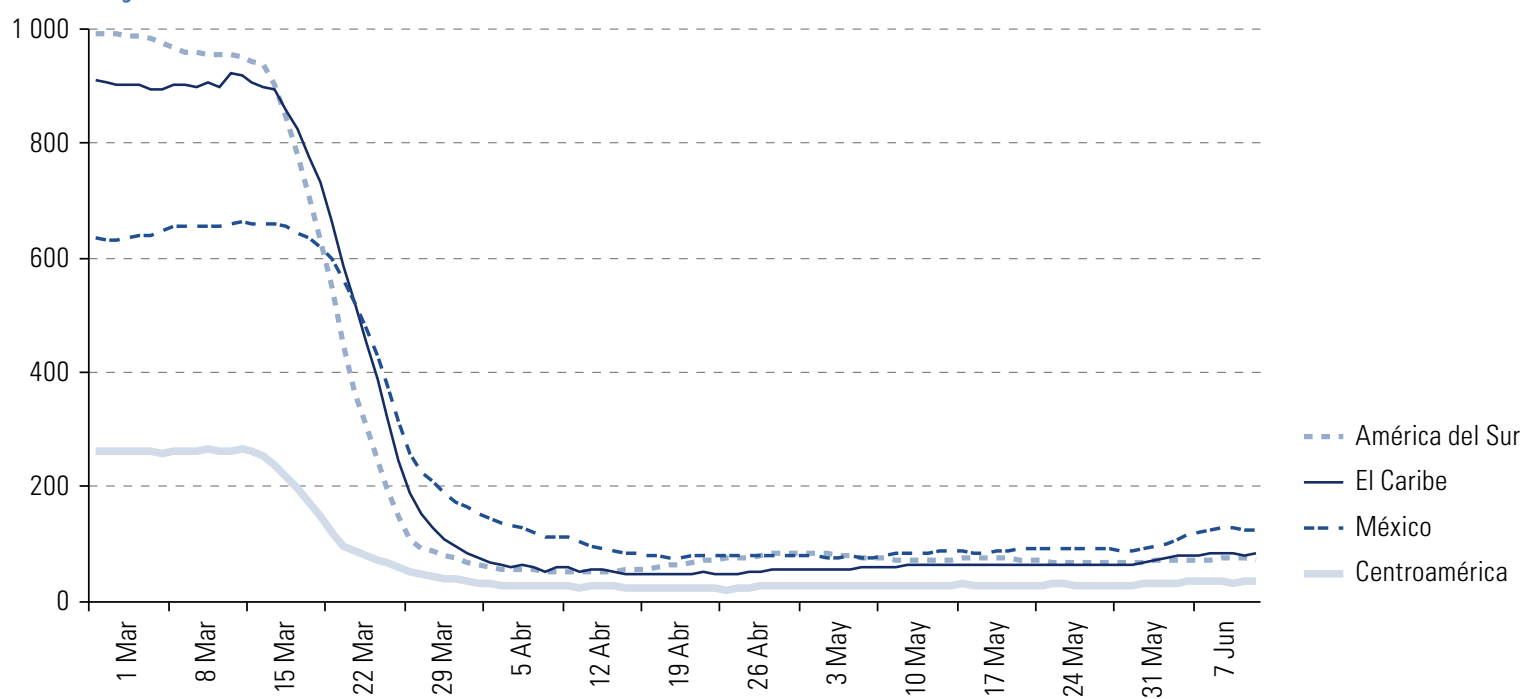

Fuente: Comisión Económica para América Latina y el Caribe (CEPAL), sobre la base de Organización de Aviación Civil Internacional (OACI), "Global COVID-19 airport status", June 2020 [online] https://www.icao.int/safety/Pages/COVID-19Airport-Status.aspx.

\section{Impactos sustanciales en las exportaciones, el PIB y el empleo}

El impacto de la marcada disminución de las llegadas de turistas en las exportaciones, el PIB y el empleo depende en gran medida de la velocidad de la recuperación en el segundo semestre. Los viajes nacionales se reanudarán antes que el turismo internacional, ya que las restricciones a los primeros se levantarán antes. Las proyecciones de la futura actividad turística deben basarse en los flujos mensuales debido a los marcados movimientos estacionales. Se simulan tres escenarios debido al alto grado de incertidumbre. Como el distanciamiento físico será la norma, se espera que la actividad turística converja en mesetas del $50 \%$, el $60 \%$ y el $70 \%$ en los escenarios pesimista, base y optimista, respectivamente. Véanse más detalles sobre el método de estimación utilizado en el recuadro 1.

Recuadro 1

Metodología para estimar las repercusiones en las exportaciones, el PIB y el empleo en América Latina y el Caribe tras la caída del turismo en 2020

Se estima que el impacto de la caída de la actividad turística en el PIB total, las exportaciones y el empleo se da de la siguiente manera:

a) Turismo entrante: i) se proyectan las llegadas mensuales de turistas internacionales hasta diciembre de 2021 utilizando las tendencias de largo plazo; ii) se utilizan datos oficiales para las llegadas entre enero y marzo de 2020 (cuando se dispone de ellos). Se proyecta que las Ilegadas de turistas internacionales disminuyan un $95 \%$ en abril de 2020 y se estanquen durante varios meses por lo menos, según los tres escenarios. Se prevé que la recuperación posterior tardará unos nueve meses, antes de llegar a mesetas del $50 \%$, el $60 \%$ y el $70 \%$, con los escenarios pesimista, de base y optimista, respectivamente (véase el cuadro y el gráfico siguientes). El impacto en las exportaciones y el PIB total se estima multiplicando la diferencia porcentual de llegadas entre la línea de base prevista y la proyección de recuperación ponderada por la proporción del turismo entrante en el las exportaciones y el PIB total, respectivamente. 
Recuadro 1 (conclusión)

Cuadro

Tres posibles escenarios para la recuperación de las llegadas de turismo internacional en 2020

\begin{tabular}{llc}
\hline $\begin{array}{l}\text { Escenario de } \\
\text { recuperación }\end{array}$ & Supuestos & $\begin{array}{c}\text { Caída prevista de la llegada } \\
\text { de visitantes en 2020 }\end{array}$ \\
\hline Optimista & $\begin{array}{l}\text { Una caída del 95\% en las llegadas continúa hasta junio de 2020. } \\
\text { Los países logran controlar la pandemia y, a partir de julio, las } \\
\text { llegadas de turistas se recuperan gradualmente hasta alcanzar } \\
\text { el 70\% de la línea de base prevista en un plazo de nueve meses. }\end{array}$ & $-52 \%$ \\
\hline De base & $\begin{array}{l}\text { La caída de las llegadas continúa hasta septiembre de 2020. La } \\
\text { recuperación comienza en octubre y las llegadas regresan al 60\% } \\
\text { de la línea de base prevista en nueve meses. }\end{array}$ & $-67 \%$ \\
\hline Pesimista & $\begin{array}{l}\text { El cierre se relaja en septiembre de 2020, pero los países no } \\
\text { logran controlar la pandemia y experimentan una segunda ola } \\
\end{array}$ & $\begin{array}{l}\text { de infecciones. Las restricciones de viaje se restablecen y duran } \\
\text { hasta diciembre de 2020. La recuperación comienza en enero de }\end{array}$ \\
\hline & $\begin{array}{l}\text { 2021 y las llegadas regresan al 50\% de la línea de base prevista } \\
\text { en nueve meses. }\end{array}$ \\
\hline
\end{tabular}

Fuente: Comisión Económica para América Latina y el Caribe (CEPAL).

Gráfico

Recuperación de las llegadas de turistas internacionales: tres escenarios posibles, 2020-2021

(Porcentaje de la línea de base prevista)

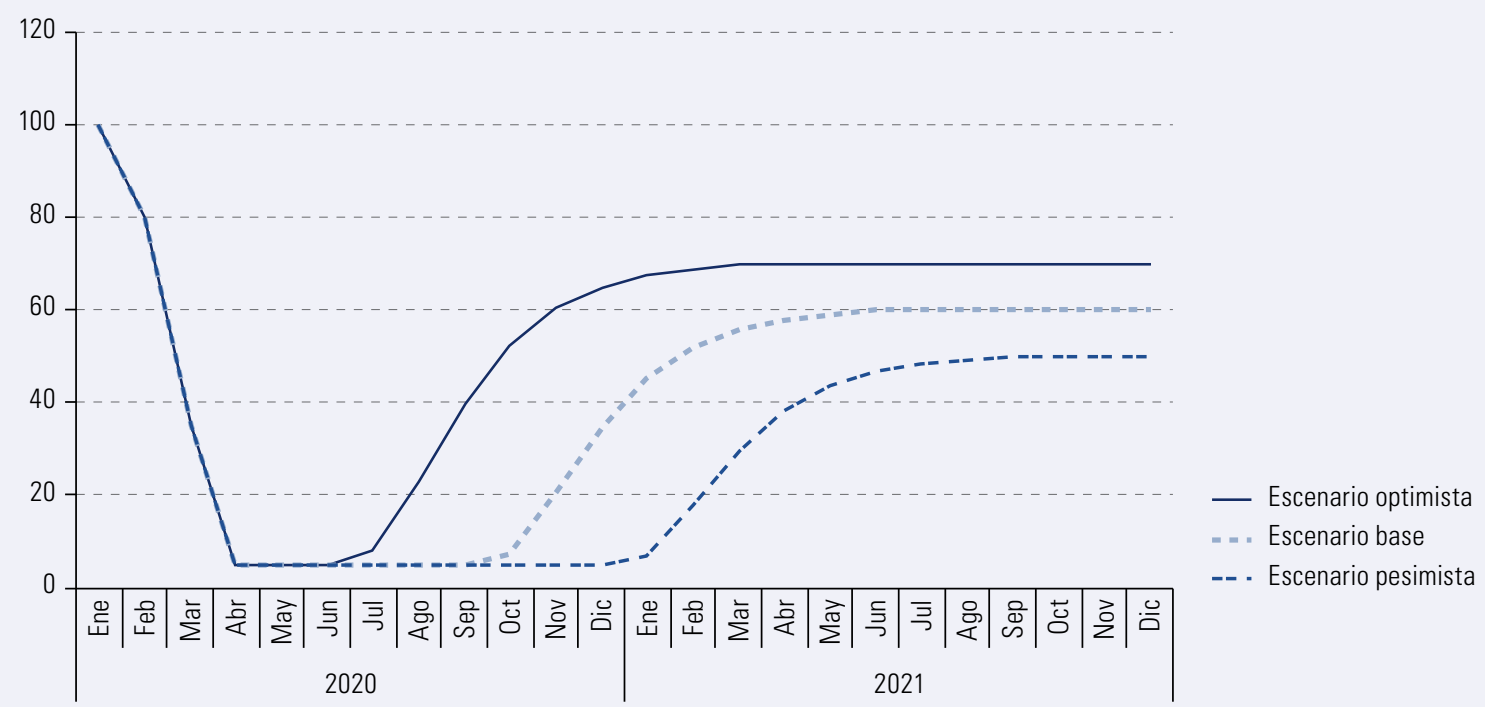

Fuente: Comisión Económica para América Latina y el Caribe (CEPAL).

b) Turismo interno: como enero y febrero fueron la temporada alta de este segmento en América del Sur, se verá menos afectado que el resto de la región. Se supone que la actividad turística interna (en términos de PIB) en el escenario base caerá un 50\% con respecto a su nivel de 2019 en América del Sur y un $60 \%$ en el resto de la región. Los escenarios optimista y pesimista suponen una caída de 20 puntos porcentuales por debajo o por encima del escenario base, respectivamente.

c) La posible pérdida de empleo se estima utilizando regresiones específicas de cada país de la relación entre las tendencias del empleo y el ciclo de crecimiento del turismo (ley de Okun), véase Kandil y otros (2014). Estas proporcionan elasticidades empleo-PIB que, a su vez, se aplican a las pérdidas del PIB. Las estimaciones de empleo son límites superiores, ya que no tienen en cuenta las medidas de mitigación aplicadas por los Gobiernos de toda la región.

Fuente: Comisión Económica para América Latina y el Caribe (CEPAL), sobre la base de M. Kandil y otros, "Labor market issues in the Caribbean: scope to mobilize employment growth", IMF Working Papers, No. WP/14/115, Washington, D.C., Fondo Monetario Internacional (FMI). 
Según estos escenarios, seis economías caribeñas podrían sufrir pérdidas de más del 50\% de su nivel de exportaciones de bienes y servicios en 2019, mientras que otras siete podrían sufrir pérdidas de más del $20 \%$. En el caso de estos países del Caribe, la pérdida de ingresos por concepto de turismo reducirá considerablemente su capacidad de importar los bienes y servicios necesarios (Coke-Hamilton, 2020). Se prevé que las pérdidas relativas por concepto de exportaciones serán menores en el caso de México y el Brasil (véase el gráfico 4), en parte porque el turismo en esos países depende mucho más de los turistas nacionales que de las llegadas internacionales. Sin embargo, las restricciones a los viajes también han paralizado los viajes internos, golpeando duramente a estos países.

Es probable que estas pronunciadas caídas en los ingresos del turismo tengan un impacto significativo en el crecimiento general del PIB. En cinco economías del Caribe, la crisis del turismo podría reducir el PIB en al menos 10 puntos porcentuales, mientras que en otras seis el PIB podría reducirse en más de 6 puntos porcentuales. Utilizando supuestos ligeramente diferentes, Mooney y Zegarra (2020) prevén que la crisis tendrá un impacto similar en el PIB de estas economías caribeñas. En todas las economías sudamericanas analizadas, se prevé que el impacto será inferior a 2 puntos porcentuales, siendo el Brasil el país que registrará la menor caída del PIB (véase el gráfico 5), debido al peso relativamente pequeño del sector en la economía general.

También se prevé que las crisis sanitaria y económica afecten negativamente al empleo en el sector del turismo, en particular si el regreso de los visitantes nacionales e internacionales es lento. Según el escenario base, que no tiene en cuenta las medidas de mitigación que están aplicando actualmente todos los Gobiernos de la región para proteger las empresas y los empleos, el empleo total podría reducirse 9 puntos porcentuales en el Caribe y 2 puntos porcentuales en América Latina (véase el gráfico 6). En el Caribe, Santa Lucía, las Bahamas, Antigua y Barbuda, Saint Kitts y Nevis y Granada pueden sufrir una pérdida total de empleo de 15 puntos porcentuales o más en el escenario pesimista. En América Latina, se espera que México sea el país que experimente la mayor caída en el empleo

Gráfico 4

América Latina y el Caribe (29 países): impacto de la disminución del turismo en las exportaciones de bienes y servicios totales en tres escenarios, 2020

(En porcentajes)

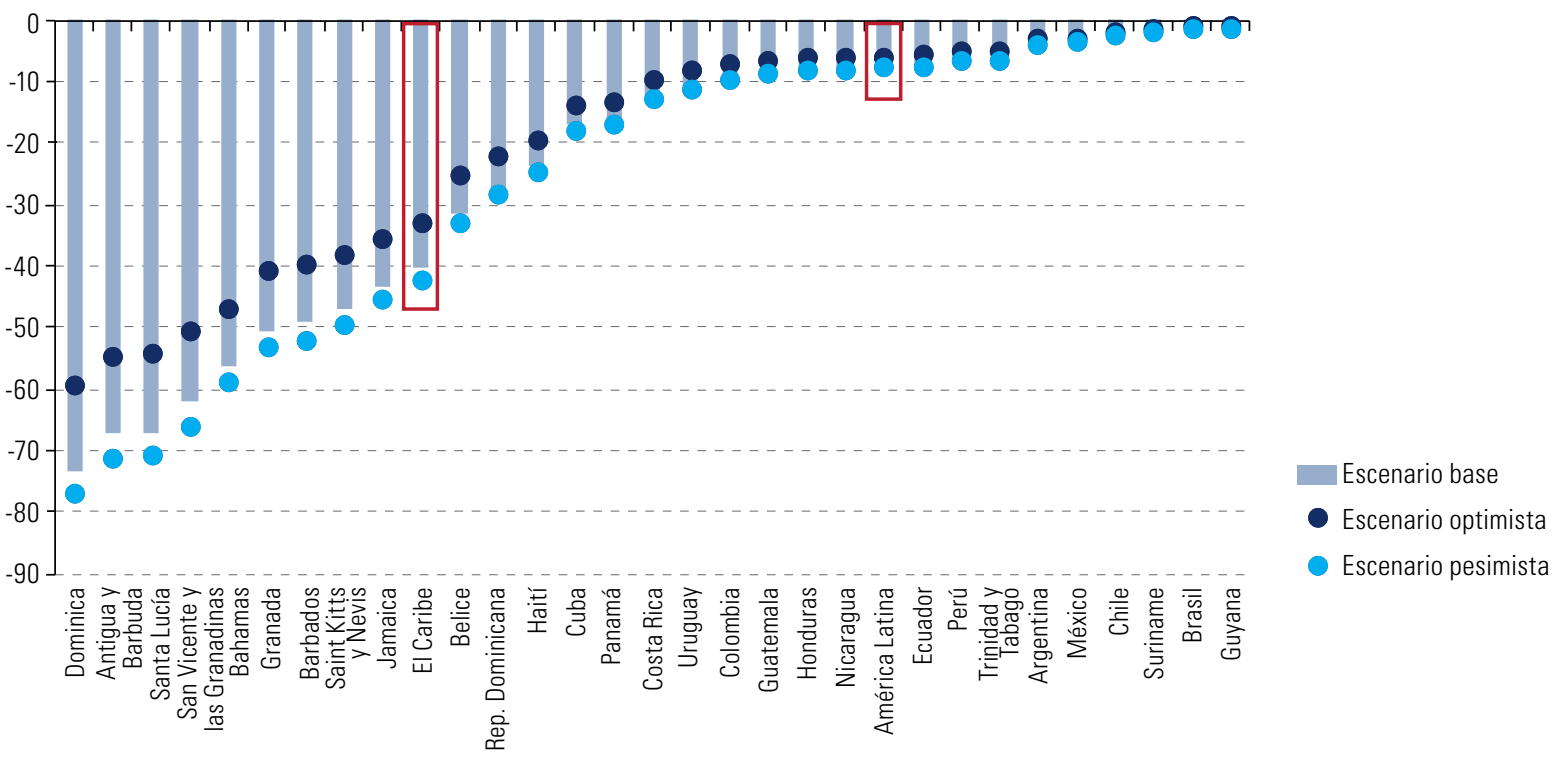

Fuente: Comisión Económica para América Latina y el Caribe (CEPAL) 


\section{Gráfico 5}

América Latina y el Caribe (28 países): impacto de la disminución del turismo en el PIB total en tres escenarios, 2020

(En puntos porcentuales)

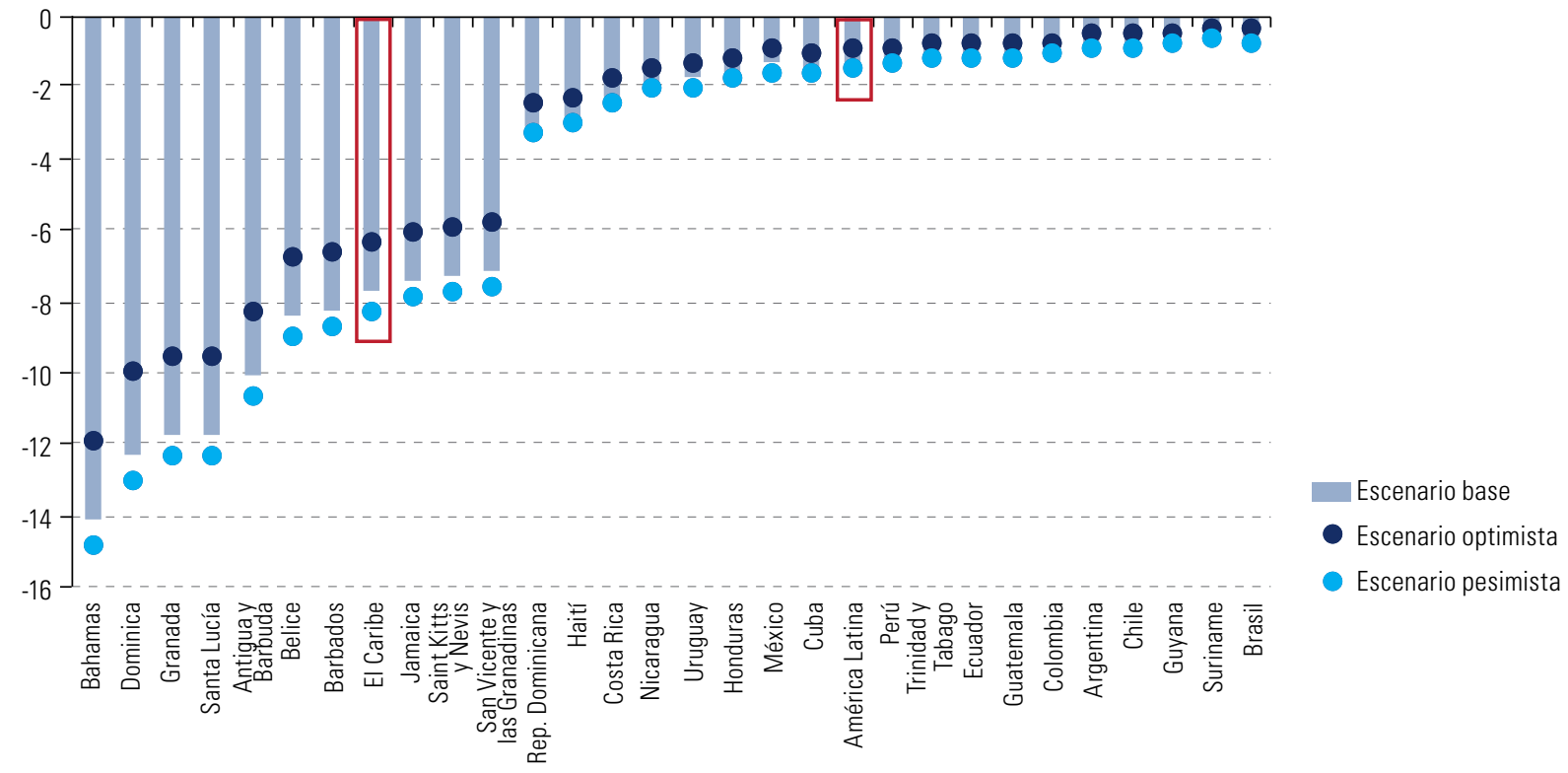

Fuente: Comisión Económica para América Latina y el Caribe (CEPAL).

Gráfico 6

América Latina y el Caribe (28 países): impacto de la disminución del turismo en el empleo total en tres escenarios, 2020 a

(En puntos porcentuales)

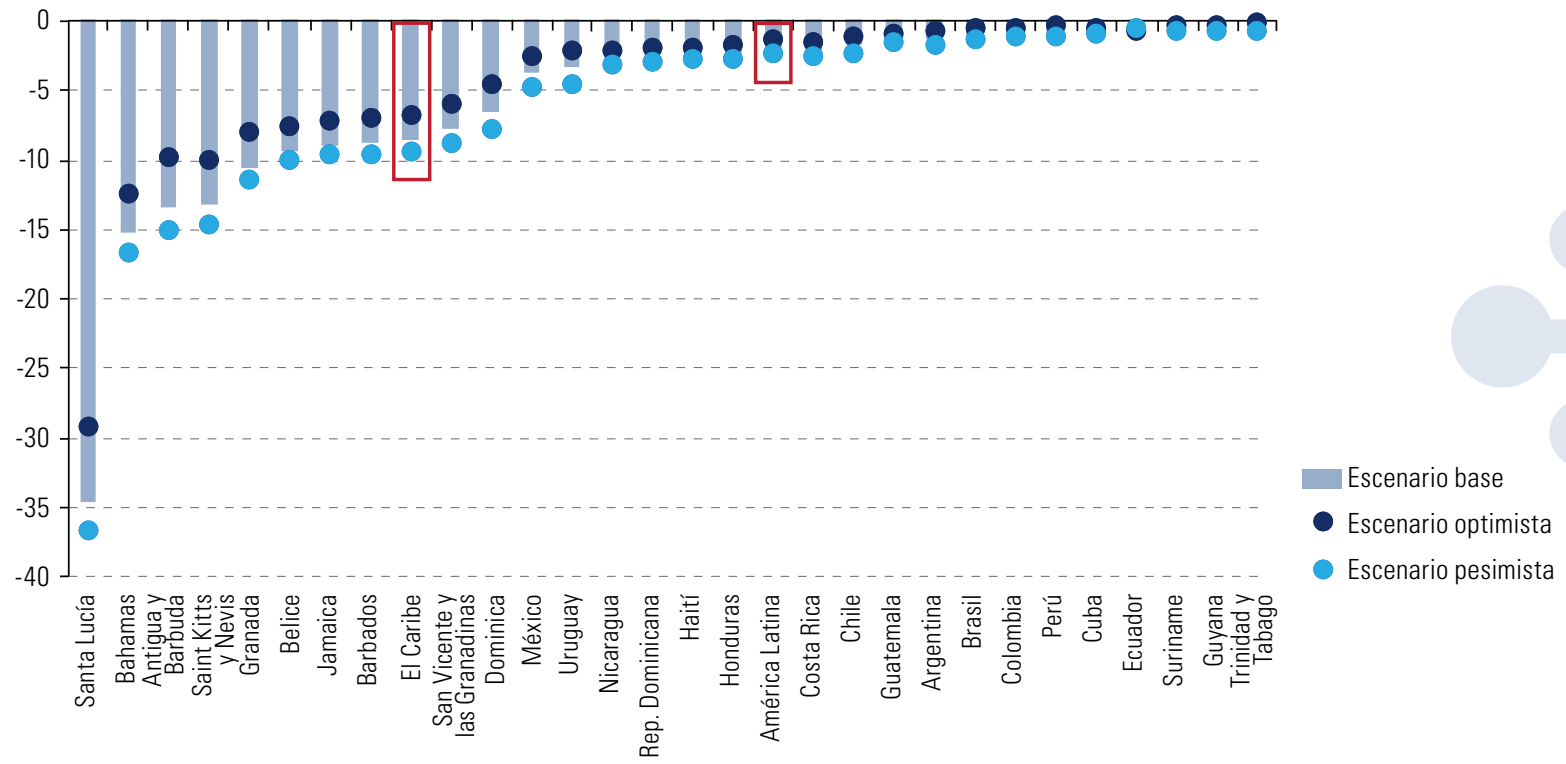

Fuente: Comisión Económica para América Latina y el Caribe (CEPAL).

a Se trata de estimaciones con un límite superior que no tienen en cuenta las medidas de mitigación aplicadas para frenar las pérdidas de empleo en el sector del turismo. 


\section{Diferentes medidas pueden mitigar el impacto de la crisis y acelerar la recuperación}

Los países de la región han adoptado medidas para mitigar los efectos económicos y sociales de la pandemia en el turismo y preparar al sector para la recuperación. Estas medidas de recuperación también deberían aumentar la diversificación del sector y su sostenibilidad social y ambiental.

\section{Protección del empleo y los ingresos de los trabajadores}

Muchos países han adoptado paquetes fiscales para prestar apoyo temporal a los ingresos de los trabajadores y propietarios de empresas del sector formal y, en algunos casos (como el Brasil), del sector informal de toda la economía. En algunos países se han introducido medidas para impedir que las empresas despidan a los trabajadores durante un determinado período (Argentina), o para permitir que los empleadores y los empleados lleguen a acuerdos sobre reducciones de la jornada laboral y la remuneración para evitar los despidos (Costa Rica). Los Gobiernos de las Bahamas, Belice y Jamaica han introducido prestaciones de desempleo temporales para los trabajadores autónomos de todos los sectores y transferencias monetarias para los trabajadores del sector del turismo, que son en su mayoría mujeres.

El empleo en el sector del turismo se caracteriza por altos niveles de informalidad y trabajo atípico, como el trabajo a tiempo parcial, temporal, ocasional, estacional, por cuenta propia e independiente. Los trabajadores que realizan tareas esenciales y los empleados de atención al cliente que trabajan en la primera línea han demostrado ser indispensables para el sector, pero la crisis también ha puesto de relieve su vulnerabilidad. Por lo tanto, los planes de recuperación deben basarse en el principio del trabajo decente para todos e incluir normas que abarquen los derechos laborales y de salud

\section{Apoyar la supervivencia de los negocios, en particular de las mipymes, a lo largo de la cadena de valor del turismo}

Entre las medidas de apoyo a la supervivencia de las empresas figuran las exenciones o prórrogas temporales del pago del impuesto sobre la renta de las sociedades (Saint Kitts y Nevis) (St Kitts \& Nevis Observer, 2020), del impuesto sobre el valor agregado (IVA) (Argentina y Colombia) y de las contribuciones a la seguridad social (Chile, Colombia y Perú); reducciones en la tasa del impuesto sobre la renta de las empresas; líneas de crédito (Ecuador) o subsidios (Guatemala) para capital de trabajo y pagos (parciales) de salarios para las mipymes y grandes empresas (Bahamas, Brasil, Colombia y Costa Rica). El Gobierno del Brasil asume el 85\% del riesgo crediticio de los préstamos a las pequeñas y medianas empresas, que deben reembolsarse en 36 meses después de un período de gracia de 6 meses. En el Ecuador y Panamá, las empresas pueden aplazar el pago de las facturas de electricidad y agua durante 12 y 4 meses, respectivamente.

Además del apoyo inmediato, es importante preparar a las mipymes del sector turístico para el futuro, específicamente mediante el fortalecimiento de las habilidades digitales. Las plataformas de reserva y los medios sociales han permitido a los proveedores de servicios turísticos interactuar directamente con sus clientes, eliminando intermediarios y reduciendo las barreras de entrada. Los conocimientos digitales también abarcan otras tecnologías innovadoras, como los grandes datos para rastrear el comportamiento de los consumidores y pronosticar la demanda, y la inteligencia artificial para diseñar experiencias únicas.

\section{Facilitar la recuperación del sector a corto plazo}

Retomar el turismo después de la pandemia será un desafío. Aunque los destinos pueden ser objetivamente seguros, muchos viajeros reacios al riesgo pueden tratar de evitar la exposición al COVID-19 y decidir no viajar en absoluto o viajar solo distancias cortas, evitando el transporte aéreo. Se pueden tomar diferentes medidas para recuperar su confianza. La más urgente es la adopción de medidas preventivas para reducir al mínimo la propagación de la enfermedad. En varios países, los ministerios de salud y de turismo, las instituciones de normas técnicas y el sector privado están colaborando en la definición de nuevos protocolos para reducir al mínimo el riesgo de contagio del COVID-19. Por ejemplo, se ha establecido un 
grupo de tareas de recuperación del turismo con participación de múltiples interesados en Antigua y Barbuda y Jamaica (Antigua News Room, 2020b; Jamaica Observer, 2020). Por su parte, el Instituto Costarricense de Turismo (ICT) ha publicado protocolos específicos para el alojamiento, el transporte, los parques nacionales y otras atracciones (ICT, 2020). El sector privado está desarrollando sus propias certificaciones sanitarias, entre las que destacan las de Accor, la Asociación Española de Normalización y Certificación (AENOR), Bureau Veritas (Iriarte Ahon, 2020) y el Consejo Mundial de Viajes y Turismo (WTTC).

El colapso de las llegadas internacionales ha puesto de relieve la necesidad de diversificación y ha llevado a varios países a promover el turismo interno para aumentar la resiliencia ante los choques. El turismo interno debería tener la doble función de reactivar la economía y reconocer la importancia del acceso a las actividades de ocio para todos. En México, los turistas nacionales representan el $83 \%$ de los ingresos del sector (INEGI, 2019). Por lo tanto, el Gobierno está promoviendo experiencias y marcas de viajes en los 32 estados del país (Secretaría de Turismo, 2020). Un enfoque alternativo para impulsar la demanda de turismo interno que se está explorando en Chile consiste en ofrecer vales o descuentos a las familias de menores ingresos o a las personas de edad.

Varios países han emprendido campañas especiales de marketing para atraer a nuevos visitantes en un futuro próximo. La Organización de Turismo del Caribe (CTO) ha planteado que la salud y el bienestar podrían ser un posible elemento de comercialización para los países de la subregión (Barbados Today, 2020). La Secretaría de Turismo de México publicó un video promocional para mostrar al país como destino turístico. Las estrategias de comercialización internacional deberían dirigirse a los grupos que tienen más probabilidades de viajar en primer lugar, como los viajeros de lujo y de negocios, que pueden adoptar medidas de distanciamiento físico con mayor facilidad y tienden a favorecer los lugares menos concurridos.

Algunas de estas estrategias pueden aplicarse más fácilmente si las empresas turísticas operan en clústers público-privados. Los clústers constituyen concentraciones geográficas de empresas, proveedores y otras instituciones interconectadas que tienen un programa estratégico para la mejora y la sofisticación de las empresas. Las empresas organizadas en clústers tienen ventajas estratégicas para hacer frente a los retos inmediatos de la pandemia y definir los programas de reactivación. En Colombia, por ejemplo, hay 18 clústers turísticos en diferentes segmentos, entre ellos el turismo de naturaleza, de negocios, de salud y cultural.

\section{Promoción de la sostenibilidad y la resiliencia del sector a mediano plazo}

Esta crisis es una oportunidad para aumentar la contribución del turismo al logro de los 17 Objetivos de Desarrollo Sostenible (ODS) de la Agenda 2030 para el Desarrollo Sostenible, al tiempo que se mejora su resiliencia a los desastres y al cambio climático. Los países solo tienen 10 años para la consecución de los ODS antes de la fecha límite de 2030. Este año marca el comienzo de la década de acción para los Objetivos de Desarrollo Sostenible, en la que el turismo es un sector clave que contribuye directa o indirectamente a todos los Objetivos, en particular el ODS 8 (trabajo decente y crecimiento económico), el ODS 13 (acción climática); el ODS 14 (vida submarina) y ODS 15 (vida de ecosistemas terrestres).

Las medidas de mitigación de la pandemia que se están aplicando actualmente en el sector del turismo podrían utilizarse para mejorar la sostenibilidad ambiental y social del sector, dado que representa aproximadamente el $5 \%$ de las emisiones mundiales de gases de efecto invernadero, de las cuales el transporte es responsable de casi tres cuartas partes, seguido del alojamiento (una quinta parte). El turismo puede causar un daño ambiental sustancial en un escenario en que todo siga igual. Sin embargo, la adopción de las medidas necesarias para ecologizar este sector podría reducir considerablemente su impacto $(O C D E, 2018)$ y atraer a turistas más conscientes del medio ambiente.

\section{Cooperación regional}

Los Gobiernos de la región deberían intensificar la colaboración para mantener las redes de transporte transfronterizas lo más abiertas posible, prestando especial atención a la facilitación del tránsito y el intercambio de las tripulaciones de los operadores de transporte (operadores de cruceros, de aerolíneas y de logística). Además, las autoridades deben abstenerse de adoptar medidas que restrinjan el tráfico en tránsito, salvo las medidas necesarias para salvaguardar la salud pública. Para ello, se deben promover soluciones digitales que limiten el contacto físico en las fronteras y protejan la salud de los trabajadores. 
Se han introducido algunas iniciativas, principalmente en la Comunidad del Caribe (CARICOM), para fomentar la cooperación y la coordinación a fin de hacer frente a la pandemia y a sus consecuencias económicas. A principios de mayo de 2020, los Jefes y Jefas de Gobierno de la CARICOM acordaron establecer un subcomité público-privado (que incluía a las líneas aéreas, los operadores de cruceros, los hoteleros y los sindicatos) para establecer los protocolos apropiados necesarios para garantizar la seguridad de los trabajadores y los visitantes cuando se reabriera el sector turístico (CARICOM Today, 2020a). Además, el Consejo Económico y Comercial de la CARICOM también recomendó que se expidieran certificados de operación a las empresas turísticas que cumplieran esos protocolos (CARICOM Today, 2020b). Deberían fortalecerse otros mecanismos de cooperación subregional, como la Secretaría de Integración Turística Centroamericana (SITCA), para hacer frente a la pandemia.

Podrían coordinarse las siguientes medidas a nivel subregional:

- Crear equipos de coordinación de crisis de múltiples países y múltiples partes interesadas para intensificar el intercambio de información sobre la salud relacionada con los viajes y otras medidas para limitar la propagación del virus. Estos equipos deberían incluir tanto a las organizaciones de turismo como a las de salud pública regionales, como la Organización Panamericana de la Salud (OPS) y el Organismo de Salud Pública del Caribe (CARPHA).

- Elaborar directrices y protocolos conjuntos para reestablecer los viajes y el turismo, que incluyan el distanciamiento físico y las restricciones en materia de higiene, el uso de equipo de protección, la desinfección dentro de los puertos y aeropuertos y a bordo de los aviones. Las organizaciones internacionales de turismo, como la OMT y el Consejo Mundial de Viajes y Turismo, están guiando estos esfuerzos.

- Fortalecer los acuerdos bilaterales o subregionales para facilitar el tránsito de los viajeros de los países signatarios.

- Promover la cooperación entre las organizaciones nacionales de turismo y las partes interesadas pertinentes en las esferas de la salud, el transporte y la inmigración, a fin de coordinar mejor las medidas para mitigar el impacto del COVID-19.

- Fomentar el intercambio de buenas prácticas entre los países para hacer frente a la crisis, incluidas la conectividad, la coordinación, las medidas nacionales de alivio y las medidas de apoyo al sector del turismo.

- Crear y aplicar un plan de recuperación de la crisis posterior a la crisis del COVID-19, que debería tratar de mejorar la capacidad turística nacional y regional; comprometer a las partes interesadas de la industria para aumentar la confianza de las empresas y los consumidores; explorar soluciones innovadoras utilizando tecnologías digitales para estimular el sector turístico; coordinar los esfuerzos de comercialización; mejorar la resiliencia del turismo; y promover el turismo sostenible e inclusivo.

- Evaluar los impactos sociales, económicos y ambientales del turismo de cruceros, y definir las normas regionales hacia el futuro.

- Promover la cooperación Sur-Sur para elaborar cuentas satélites de turismo y normalizar los datos sobre el turismo

\section{Bibliografía}

Antigua News Room (2020a), "LIAT further extends suspension of passenger services", 14 de mayo [en línea] https://antiguanewsroom.com/liat-further-extends-suspension-passengers-services/.

(2020b), "Tourism industry stakeholders meet virtually to discuss plans for Antigua \& Barbuda tourism re-opening", 9 de mayo [en línea] https://antiguanewsroom.com/tourism-industry-stakeholders-meetvirtually-to-discuss-plans-for-antigua-barbuda-tourism-re-opening/.

Banco Mundial (2017), Women and Tourism: Designing for Inclusion, Washington, D.C.

Barbados Today (2020), "CTO: Brace for '25-year' tourism setback", 1 de mayo [en línea] https://barbadostoday. bb/2020/05/01/cto-brace-for-25-year-tourism-setback/.

BCCR (Banco Central de Costa Rica) (2018), "Cuenta Satélite de Turismo" [en línea] https://www.bccr.fi.cr/ seccion-indicadores-economicos/cuenta-sat\%C3\%A9lite-de-turismo.

CARICOM Today (2020b), "STATEMENT - Tenth Special Emergency Meeting of the Conference of Heads of Government of the Caribbean Community (CARICOM), Via Video Conference, 5 May 2020", 11 de mayo [en línea] https:// today.caricom.org/2020/05/07/statement-tenth-special-emergency-meeting-of-the-conference-of-headsof-government-of-the-caribbean-community-caricom-via-video-conference-5-may-2020/. 
(2020b), "CARICOM's Trade and Economic Council approves strategy for the re-opening of regional economies", 12 de mayo [en línea] https://today.caricom.org/2020/05/07/caricoms-trade-andeconomic-council-approves-strategy-for-the-re-opening-of-regional-economies/.

Coke-Hamilton, P. (2020), "Impact of COVID-19 on tourism in small island developing States", Conferencia de las Naciones Unidas sobre Comercio y Desarrollo (UNCTAD), 24 de abril [en línea] https://unctad. org/en/pages/newsdetails.aspx? OriginalVersionID=2341.

Dini, M. y G. Stumpo (coords.) (2018), "Mipymes en América Latina: un frágil desempeño y nuevos desafíos para las políticas de fomento", Documentos de Proyectos (LC/TS.2018/75), Santiago, Comisión Económica para América Latina y el Caribe (CEPAL).

ICT (Instituto Costarricense de Turismo) (2020), "Material de apoyo coronavirus (sector turismo)" [en línea] https://www.ict.go.cr/es/servicios-institucionales/material-de-apoyo-coronavirus-sector-turismo.html.

INEGI (Instituto Nacional de Estadística y Geografía) (2019), "Cuenta Satélite del Turismo de México" [en línea] https://www.datatur.sectur.gob.mx/SitePages/ProductoDestacado3.aspx.

Iriarte Ahon, E. (coord.) (2020), "Informe: lineamientos y herramientas que, bajo un enfoque innovador y con énfasis en la transformación digital, buscan promover el sector turismo en el corto, mediano y largo plazo tras el Estado de Emergencia Nacional por el COVID-19, considerando los nuevos cambios de paradigmas del viajero nacional e internacional, que exigirá que los prestadores de servicios turísticos se reinventen para sobrevivir", mayo, inédito.

Jamaica Observer (2020), "Tourism minister confirms opposition seat on COVID-19 Tourism Recovery Taskforce", 24 de abril [en línea] http://www.jamaicaobserver.com/latestnews/Tourism_minister_ confirms_opposition_seat_on_COVID-19.

Mooney, H. y M. A. Zegarra (2020), "COVID-19: tourism-based shock scenarios for Caribbean countries", Banco Interamericano de Desarrollo (BID), 16 de marzo [en línea] https://blogs.iadb.org/caribbean-dev-trends/ en/covid-19-tourism-based-shock-scenarios-for-caribbean-countries/.

OCDE (Organización de Cooperación y Desarrollo Económicos) (2018), "Analysing megatrends to better shape the future of tourism", OECD Tourism Papers, $N^{\circ} 2018 / 02$, París, OECD Publishing.

OMT (Organización Mundial del Turismo) (2019), Global Report on Women in Tourism - Second Edition, Madrid. (2020), World Tourism Barometer, vol. 18, № 2, mayo.

Panetta, G. (2020), "Cruise ship bookings for 2021 are already on the rise despite multiple COVID-19 outbreaks", Business Insider, 12 de abril [en línea] https://www.businessinsider.com/cruise-ship-bookings-areincreasing-for-2021-despite-coronavirus-2020-4.

Secretaría de Turismo (2020), 'Presenta Miguel Torruco la estrategia digital para la contención de crisis del sector turístico", 8 April [en línea] https://www.gob.mx/sectur/prensa/presenta-miguel-torruco-laestrategia-digital-para-la-contencion-de-crisis-del-sector-turistico.

St Kitts \& Nevis Observer (2020), "COVID-19: Government's 17-Point Stimulus Package Includes Reducing Corporate and Business Tax", 25 de marzo [en línea] https://www.thestkittsnevisobserver.com/covid19-governments-17-point-stimulus-package-includes-reducing-corporate-and-business-tax/

Este documento es parte de un conjunto de informes elaborados por la Comisión Económica para América Latina y el Caribe (CEPAL) sobre la evolución y los efectos de la pandemia del COVID-19 en América Latina y el Caribe. Fue preparado por la División de Comercio Internacional e Integración, a cargo de Mario Cimoli, en colaboración con las sedes subregionales de la CEPAL para el Caribe en Puerto España y en México y su oficina nacional en Brasilia, bajo la coordinación general de Alicia Bárcena, Secretaria Ejecutiva de la CEPAL.

Copyright (C) Naciones Unidas, 2020

C Comisión Económica para América Latina y el Caribe (CEPAL)

Economic Commission for Latin America and the Caribbean (ECLAC)

www.cepal.org 